This item was submitted to Loughborough's Research Repository by the author.

Items in Figshare are protected by copyright, with all rights reserved, unless otherwise indicated.

\title{
Surface thermodynamics reconsidered. Derivation of the Gokhshtein relations from the Gibbs potential and a new approach to surface stress
}

PLEASE CITE THE PUBLISHED VERSION

http://dx.doi.org/10.1007/s10008-013-2287-9

\section{PUBLISHER}

(c) Springer-Verlag

VERSION

AM (Accepted Manuscript)

\section{PUBLISHER STATEMENT}

This work is made available according to the conditions of the Creative Commons Attribution-NonCommercialNoDerivatives 4.0 International (CC BY-NC-ND 4.0) licence. Full details of this licence are available at: https://creativecommons.org/licenses/by-nc-nd/4.0/

\section{LICENCE}

CC BY-NC-ND 4.0

\section{REPOSITORY RECORD}

Fletcher, Stephen. 2019. "Surface Thermodynamics Reconsidered. Derivation of the Gokhshtein Relations from the Gibbs Potential and a New Approach to Surface Stress". figshare. https://hdl.handle.net/2134/21087. 


\title{
Surface Thermodynamics Reconsidered. Derivation of the Gokhshtein Relations from the Gibbs Potential; and a New Approach to Surface Stress.
}

\author{
Stephen Fletcher
}

\begin{abstract}
Prof S. Fletcher, Department of Chemistry, Loughborough University, Ashby Road, Loughborough, Leicestershire LE11 3TU, UK.

E-mail: stephen.fletcher@lboro.ac.uk.
\end{abstract}

Submitted 22 May 2013. Revised 24 September 2013,

Accepted 05 October 2013, Published online 24 November 2013

(C) 2013 Springer-Verlag Berlin Heidelberg.

Cite as:

Fletcher, Stephen. Surface thermodynamics reconsidered. Derivation of the Gokhshtein relations from the Gibbs potential and a new approach to surface stress. Journal of Solid State Electrochemistry 18, no. 5: 1231-1238 (2014).

\section{Dedication}

Dedicated to the memory of Professor Vladimir Sergeevich Bagotsky (1920-2012)

Keywords

Thermodynamics, Surface Tension; Surface Stress; Maxwell Relations;

Electrochemical Potential; Gokhshtein Relations

\begin{abstract}
The terminology and definition of surface tension are discussed. In particular, the surface tension is defined as the partial derivative of the surface excess Gibbs energy with respect to an infinitesimal increment of surface area at constant temperature and pressure. The surface tension is also formulated as the sum of a stress-free component and a stress-containing component. The stress-containing component is defined as the surface stress. Finally, the case of charged surfaces is analysed, and the Gokhshtein relations are derived from the Gibbs potential, in the special case that the electrode/solution interface is ideally polarizable.
\end{abstract}




\section{Introduction}

Vladimir Sergeevich Bagotsky had a lifelong interest in electrochemistry and electrochemical thermodynamics [1]. His first monograph Kinetics of Electrode Processes was published with A. N. Frumkin, Z. A. lofa, and B. N. Kabanov in 1952; his final work Fuel Cells: Problems and Solutions appeared in 2009 [2,3]. In between, he published more than 400 technical papers on electrochemical topics, many concerned with advanced power sources. Meanwhile, his textbook Fundamentals of Electrochemistry provided students with an authoritative introduction to electrochemical science [4]. His passing marks the end of an era.

During much of Bagotsky's lifetime, the "cold war" hampered the normal exchange of scientific ideas between Russia and the West, so that developments on one side were sometimes completely missed by the other. One such area was surface thermodynamics, for which many important Russian developments are only now coming to light [5]. For example, the surface tension of solids was considered by A. Ya. Gokhshtein as long ago as 1976, but his book has never been translated into English [6]. Remarkably, Gokhshtein introduced two new thermodynamic relations that have still not been thoroughly assessed by western researchers.

In the present work, I derive the Gokhshtein relations from the Gibbs potential. My hope is that this will help clarify the logical status of the Gokhshtein relations, and incidentally show how they connect with the corresponding Maxwell relations.

\section{The Measurement of Surface Tension}

Surface tension is so-called because a tensile force must be applied to a surface to balance its natural tendency to contract. Experimentally, an obvious way of estimating the surface tension $(\gamma)$ is to measure the partial derivative of the work-offorming-the-surface $(w)$ with respect to the surface area $(A)$, whilst holding certain variables (such as temperature and pressure) constant.

$$
\gamma_{\exp }=\left(\frac{\partial w(A)}{\partial A}\right)_{T, P}
$$

This approach suffers from a number of defects, however [7]. Most importantly, the measured value of $\gamma$ will depend on the precise method by which the surface area is changed. Friction forces may also be included inadvertently in the measurement. Further, there may also be configurationally trapped states (as in glasses) which do not relax on the timescale of the experiment (or even on the timescale of the Universe!). 
To avoid these complications, surface scientists prescribe a special way of changing $A$ so that friction forces, trapped configurations, and multiple pathways are avoided. What they do is focus on (thought-) experiments in which the work of forming $A$ is absolutely minimal at every stage, so that the value of $\gamma$ depends only on the value of $A$ and not at all on the path by which $A$ is reached. In the jargon of thermodynamics, this is called the "reversible" path. Further, if the temperature and pressure are held constant while this hypothetical experiment is performed, then the work $w$ may be replaced by the surface excess Gibbs energy $G$,

$$
\gamma(A)=\left(\frac{\partial G(A)}{\partial A}\right)_{\mathrm{T}, \mathrm{P}}
$$

This is the definition of surface tension found in many reviews and textbooks. (See, for example, Eq. (4.47) of R. Defay and I. Prigogine [8]) It is, strictly speaking, the definition of the reversible surface tension [although no-one seems to use this terminology] and it requires a state of thermodynamic equilibrium at all values of $A$.

To summarize: the use of the surface excess Gibbs energy $G$ rather than the work $w$ compels $\gamma$ to be a function of state. This has advantages and disadvantages. An advantage is that, by restricting attention to changes in $G$, the value of the reversible surface tension $\gamma$ depends only on the value of $A$ and not at all on the path by which $A$ is reached. But a disadvantage is that it leaves open the question of how the minimum Gibbs energy is actually achieved in a real system. For example, the minimum Gibbs energy might be achieved by adding new surface atoms or by stretching apart existing surface atoms (or some combination of both). Which mechanism of expanding the surface area actually occurs will depend on the geometry, constitution, and boundary conditions of the system. On a solid nanocrystal, for example, one might expect some stretching of low-energy crystal faces as a trade-off against the extension of high-energy crystal faces. Indeed, stretching might well be central to the functioning of these catalytic surfaces.

\section{The Terminology of Surface Tension}

In general, the term "surface tension" (interfacial tension) is not the name of a single parameter. Actually it is the generic name for a large set of parameters that describe how various different thermodynamic potentials vary with small changes of surface area, given certain constraints on a system. These constraints (constant temperature, constant pressure, constant volume...) may be applied externally or may arise spontaneously inside the system due to unavoidable couplings between parameters. (In the latter case, the couplings are sometimes referred to as "equations of state"). Likewise, the term "surface energy" is not the name of a single parameter. It is the generic name for a large set of different parameters that describe how different 
thermodynamic potentials vary with large changes of surface area, given certain constraints on the system.

In what follows we arbitrarily restrict attention to one thermodynamic potential (the Gibbs potential) and two external constraints (constant temperature and constant pressure).

At constant temperature and pressure, and in the absence of a surface excess electric charge, the thermodynamics of surfaces can be treated as a purely mechanical problem. Indeed, under these circumstances, and assuming an isotropic solid, the surface excess Gibbs energy $G$ is a function of one variable only, namely the surface area $A$. Thus

$$
G=G(A)
$$

The existence of this simple relationship suggests that it might be useful to define a surface excess Gibbs energy density $\hat{G}(A)$ as the quotient

$$
\widehat{G}(A)=\frac{G(A)}{A}
$$

(where the surface area $A$ refers to a flat surface).

The motivation for this approach is the hope is that, in any given system, $\hat{G}(A)$ will turn out to be a surface area-independent (intensive) parameter characteristic of the surface as a whole. Unfortunately, this hope will certainly be forlorn if elastic forces exist inside the surface. For this reason, the presence or absence of elastic forces must be determined by experiment.

Before proceeding to the theory, we note that the macroscopic quotient referred to as the "surface excess Gibbs energy density" has the same units $\left(\mathrm{J} \mathrm{m}^{-2}\right)$ as the microscopic derivative referred to as the "surface tension" (interfacial tension). It is therefore easy to get them confused, and, to avoid this, we now briefly digress to see how they can be distinguished.

\section{The Definition of Surface Tension}

In the present work, the surface tension (which, in the most general case, may be surface area-dependent) is defined as the partial derivative of the surface excess Gibbs energy (J) with respect to an infinitesimal increment of surface area $\left(\mathrm{m}^{2}\right)$ at constant $T$ and $P$, as given in Eq.(2). As a partial derivative of a thermodynamic potential with respect to an extensive variable, the surface tension is a "generalized 
force". Like a classical force, this generalized force must always be balanced by an opposing force at equilibrium, usually a body force. Further, since the surface excess Gibbs energy arises from the difference in Gibbs energy between the total system before interface formation, and the total system after interface formation, the surface tension must be considered to arise (to a greater or lesser extent) from the interactions of all the particles in the system.

By substitution we immediately find that

$$
\gamma(A)=\left(\frac{\partial G(A)}{\partial A}\right)_{\mathrm{T}, \mathrm{P}}=\left(\frac{\partial[A \hat{G}(A)]}{\partial A}\right)_{\mathrm{T}, \mathrm{P}}=\left[\hat{G}(A)+A\left(\frac{\partial \widehat{G}(A)}{\partial A}\right)\right]_{\mathrm{T}, \mathrm{P}}
$$

where $\widehat{G}(A)$ is the surface excess Gibbs energy density. From Eq. (5) we note that, under the specified constraints of constant temperature and pressure, the microscopic derivative "surface tension" $\gamma(A)$ depends upon two non-independent terms containing the macroscopic quotient $\widehat{G}(A)$. The first term is $\widehat{G}(A)$ itself, while the second term involves the derivative of $\widehat{G}(A)$ with respect to surface area.

We emphasize that $\hat{G}(A)$ is not necessarily a constant. However, it is also obvious by inspection that a great simplification is achieved if

$$
\widehat{G}(A)=\text { constant }
$$

In that case

$$
\left(\frac{\partial \widehat{G}}{\partial A}\right)_{\mathrm{T}, \mathrm{P}}=0
$$

and the surface tension $\gamma$ and the surface excess Gibbs energy density $\hat{G}$ become identical.

Consider, however, what happens when the surface tension $\gamma$ is a function of surface area $A$. Then by analogy with Young's modulus we can define a modulus of surface tension $E(A)$ such that

$$
E(A)=A\left(\frac{\partial \gamma}{\partial A}\right)_{\mathrm{T}, \mathrm{P}}
$$


This parameter measures the mechanical stiffness of a surface. If it has a finite value then we are justified in concluding that the surface contains at least one elastic force, in addition to the normal contractive force due to the termination of the bulk phase.

The possibility of a finite modulus of surface tension means that two different definitions of surface tension are possible. One definition includes the possible existence of elasticity, and the other doesn't. For avoidance of ambiguity, let us state here that we consider the surface tension to be the coefficient

$$
\gamma_{\mathrm{T}, \mathrm{P}}
$$

(DEFINITION 1)

which applies universally to both solids and liquids, and does allow for the inclusion of elastic behaviour. We do NOT consider surface tension to be the coefficient

$$
\gamma_{\mathrm{T}, \mathrm{P},(\partial \gamma / \partial \mathrm{A}=0)}
$$

(DEFINITION 2)

which applies only to ideal liquids. In the case of DEFINITION 1, "stretching" or "compression" of the surface is permitted by the formalism, such that the change of surface area may result in a change of surface excess Gibbs energy density $\hat{G}$. In the case of DEFINITION 2, elastic stretching or elastic compression of the surface is not permitted by the formalism, so the change of surface area must be such that the new area has precisely the same surface properties as the old area. In physical terms, this means that DEFINITION 2 applies only to ideal (zero-modulus-of-surfacetension) liquids.

In a well-known paper [9], Shuttleworth derived an equation analogous to Eq. (5), except that he used the surface excess Helmholtz energy density $\widehat{F}$, rather than $\hat{G}$. Interestingly, he noted that his definition of the surface tension was true "no matter what the manner of deformation of the surface". [That is, he was also using DEFINITION 1.]

Although Eq. (5) is merely the mathematical elaboration of one possible definition of surface tension, it does emphasize an important physical fact - the surface tension $\gamma$ (according to DEFINITION 1) is not necessarily equal to the surface excess Gibbs energy density $\hat{G}$. As discussed above, the values of $\gamma$ and $\hat{G}$ coincide only when the surface excess Gibbs energy density $\hat{G}$ is independent of surface area. Although independence is assured in the case of a hypothetical liquid surface lacking longrange order, it may happen that both $\gamma$ and $\hat{G}$ are functions of $A$ on real liquid surfaces. Indeed, this is commonly the case in the presence of lipid films, and on solid surfaces.

\section{Surface Stress}


In the expansion of $\gamma(A)$ given by Eq. (5), it may be observed that both terms on the right-hand side are functions of area $A$. Here we seek a different expansion of $\gamma(A)$ of the form

$$
\gamma(A)=\widehat{G}_{1}+\widehat{G}_{2}(A)
$$

such that the first term $\widehat{G}_{1}$ is independent of $A$, and the second term $\widehat{G}_{2}(A)$ is dependent on $A$. Since surface stress involves the deformation of surface, we identify $\hat{G}_{1}$ as the stress-free component of the surface tension, and $\widehat{G}_{2}(A)$ as the stress-containing component of the surface tension.

To begin, we first expand $\widehat{G}(A)$ as a MacLaurin series

$$
\hat{G}(A)=\hat{G}(0)+A \hat{G}^{\prime}(0)+A^{2} \frac{\hat{G}^{\prime \prime}(0)}{2 !}+\ldots+A^{n} \frac{\widehat{G}^{(n)}(0)}{n !}+\ldots
$$

Then we insert the series into Eq. (5) and collect terms, so that

$$
\gamma(A)=\hat{G}(0)+A\left[\hat{G}^{\prime}(0)+\hat{G}^{\prime}(A)\right]+\sum_{n=2}^{\infty} A^{n} \frac{\hat{G}^{(n)}(0)}{n !}
$$

[Note that $\gamma(A)$ is assumed to be isotropic, which removes the need for treating it as a tensor.] Now retaining only terms up to first order in $A$, we obtain the approximation

$$
\gamma(A) \approx \hat{G}(0)+A\left[\hat{G}^{\prime}(0)+\hat{G}^{\prime}(A)\right]
$$

Finally, comparing this result term-by-term with Eq. (9) reveals that $\widehat{G}_{1}=\hat{G}(0)$ and that

$$
\widehat{G}_{2}(A) \approx A\left[\hat{G}^{\prime}(0)+\hat{G}^{\prime}(A)\right]
$$

Since $\hat{G}_{2}(A)$ is the stress-containing component of the surface tension, we here identify it as the surface stress $\tau(A)$.

By differentiation of Eq. (9) we readily find that

$$
E(A)=A\left(\frac{\partial \gamma}{\partial A}\right)_{\mathrm{T}, \mathrm{P}}=A\left(\frac{\partial \tau}{\partial A}\right)_{\mathrm{T}, \mathrm{P}}
$$


This proves that, to first order in $A$, the modulus of surface tension and the modulus of surface stress are equal. However, the surface tension and the surface stress are not themselves equal, because they differ by a constant of integration equal to the stress-free component of the surface tension $\widehat{G}_{1}$. Whether $\widehat{G}_{1}$ and $\widehat{G}_{2}(A)$ can be partitioned experimentally is an open question.

The validity of retaining only the linear terms in the MacLaurin expansion is also an open question, although comparison with Hooke's Law suggests that such an approximation might be reasonable.

\section{Charged Surfaces}

The presence of a surface excess electric charge greatly complicates the thermodynamic analysis of surfaces. For the case of a charged surface it is necessary to take into account the fact that the surface excess Gibbs energy $G$ is now a function of two variables, the surface area $A$ and the surface excess charge $Q$. Thus

$$
G=G(A, Q)
$$

We assume that, in general, the surface area $A$ and the surface excess charge $Q$ may (or may not) be coupled, depending on the type of perturbation that the surface is subjected to. For example, if the surface area is increased at constant surface excess charge density $q$ (a process similar to adding an extra tile to a set of preexisting tiles) then $A$ and $Q$ are linearly related; but if an existing tile is mechanically stretched at fixed $Q$, then $A$ and $Q$ may not be related at all. To accommodate these extreme possibilities within one mathematical framework, we must write the surface excess charge density either explicitly as

$$
q=q(A, Q)
$$

or implicitly as

$$
A=A(q, Q)
$$

We then evaluate the total derivatives

$$
\mathrm{d} G=\left(\frac{\partial G}{\partial A}\right)_{\mathrm{Q}} \mathrm{d} A+\left(\frac{\partial G}{\partial Q}\right)_{\mathrm{A}} \mathrm{d} Q
$$


and

$$
\mathrm{d} A=\left(\frac{\partial A}{\partial q}\right)_{\mathrm{Q}} \mathrm{d} q+\left(\frac{\partial A}{\partial Q}\right)_{\mathrm{q}} \mathrm{d} Q
$$

Substituting Eq. (19) into Eq. (18) then gives the expression

$$
\mathrm{d} G=\left(\frac{\partial G}{\partial q}\right)_{\mathrm{Q}} \mathrm{d} q+\left(\frac{\partial G}{\partial A}\right)_{\mathrm{Q}}\left(\frac{\partial A}{\partial Q}\right)_{\mathrm{q}} \mathrm{d} Q+\left(\frac{\partial G}{\partial Q}\right)_{\mathrm{A}} \mathrm{d} Q
$$

Although complicated, this result is exact. It can be simplified in various ways, and some particular cases can be derived from it by taking appropriate limits. For example, at constant surface excess charge $Q$ we have $\mathrm{d} Q=0$ and

$$
\mathrm{d} G=\left(\frac{\partial G}{\partial q}\right)_{\mathrm{Q}} \mathrm{d} q
$$

Forming the derivative with respect to surface area at constant $Q$ then yields

$$
\left(\frac{\partial G}{\partial A}\right)_{\mathrm{Q}}=\left(\frac{\partial G}{\partial A}\right)_{\mathrm{Q}}\left(\frac{\partial A}{\partial q}\right)_{\mathrm{Q}}\left(\frac{\partial q}{\partial A}\right)_{\mathrm{Q}}=\gamma_{\mathrm{Q}}
$$

Alternatively, at constant surface excess charge density $q$ we have $d q=0$ and

$$
\mathrm{d} G=\left(\frac{\partial G}{\partial A}\right)_{\mathrm{Q}}\left(\frac{\partial A}{\partial Q}\right)_{\mathrm{q}} \mathrm{d} Q+\left(\frac{\partial G}{\partial Q}\right)_{\mathrm{A}} \mathrm{d} Q
$$

Forming the derivative with respect to surface area at constant $q$ yields

$$
\begin{gathered}
\left(\frac{\partial G}{\partial A}\right)_{\mathrm{q}}=\left(\frac{\partial G}{\partial A}\right)_{\mathrm{Q}}\left(\frac{\partial A}{\partial Q}\right)_{\mathrm{q}}\left(\frac{\partial Q}{\partial A}\right)_{\mathrm{q}}+\left(\frac{\partial G}{\partial Q}\right)_{\mathrm{A}}\left(\frac{\partial Q}{\partial A}\right)_{\mathrm{q}} \\
=\left(\frac{\partial G}{\partial A}\right)_{\mathrm{Q}}+\left(\frac{\partial G}{\partial Q}\right)_{\mathrm{A}}\left(\frac{\partial Q}{\partial A}\right)_{\mathrm{q}} \\
=\gamma_{\mathrm{Q}}+\left(\frac{\partial G}{\partial Q}\right)_{\mathrm{A}}\left(\frac{\partial Q}{\partial A}\right)_{\mathrm{q}}
\end{gathered}
$$




$$
=\gamma_{\mathrm{q}}
$$

Finally, combining the above results, we readily obtain

$$
\begin{aligned}
\gamma_{\mathrm{q}} & =\gamma_{\mathrm{Q}}+\left(\frac{\partial G}{\partial Q}\right)_{\mathrm{A}}\left(\frac{\partial Q}{\partial A}\right)_{\mathrm{q}} \\
& =\gamma_{\mathrm{Q}}+E q_{\mathrm{A}}
\end{aligned}
$$

Here $\gamma_{Q}$ is the surface tension measured at constant charge, and $\gamma_{\mathrm{q}}$ is the surface tension measured at constant charge density. $E$ is the electrode potential measured from the point of zero charge, and $q_{\mathrm{A}}$ is the charge density.

\section{The Surface Analogs of the Maxwell Relations}

Relations between the second derivatives of thermodynamic potentials are called Maxwell relations. Due to the large number of possible thermodynamic potentials there are a large number of Maxwell relations. Even for a system having just two degrees of freedom there are 4 thermodynamic potentials $F, G, H$, and $U$, and four Maxwell relations. For three degrees of freedom this rises to 8 thermodynamic potentials, and for four degrees of freedom there are 16 thermodynamic potentials.

In practice, it turns out that most Maxwell relations are of little use. A few, however, are exceptionally useful.

For two degrees of freedom, for a surface that is subject to change by hydrostatic work only (i.e. $P V$ work only), the differential forms of $F, G, H$, and $U$ are

$$
\begin{aligned}
& \mathrm{d} F=-S \mathrm{~d} T-P \mathrm{~d} V \\
& \mathrm{~d} G=-S \mathrm{~d} T+V \mathrm{~d} P \\
& \mathrm{~d} H=T \mathrm{~d} S+V \mathrm{~d} P \\
& \mathrm{~d} U=T \mathrm{~d} S-P \mathrm{~d} V
\end{aligned}
$$

These equations apply to any surface having a constant surface area $A$ and constant number of adsorbed charged species $N$.

Because the potentials $F, G, H$, and $U$ are functions of state, their differentials are exact. And as we would expect, each potential has a different pair of variables as its natural variables: 


$$
\begin{aligned}
& F=F(T, V) \\
& G=G(T, P) \\
& H=H(S, P) \\
& U=U(S, V)
\end{aligned}
$$

If any one of these potentials is known explicitly in terms of its natural variables then the system is fully defined. Further, if we differentiate a second time, we get the surface analogs of the bulk phase Maxwell relations

$$
\begin{gathered}
\frac{\partial^{2} F}{\partial T \partial V}=-\left(\frac{\partial P}{\partial T}\right)_{\mathrm{V}}=-\left(\frac{\partial S}{\partial V}\right)_{\mathrm{T}} \\
\frac{\partial^{2} G}{\partial T \partial P}=+\left(\frac{\partial V}{\partial T}\right)_{\mathrm{P}}=-\left(\frac{\partial S}{\partial P}\right)_{\mathrm{T}} \\
\frac{\partial^{2} H}{\partial S \partial P}=+\left(\frac{\partial V}{\partial S}\right)_{\mathrm{P}}=+\left(\frac{\partial T}{\partial P}\right)_{\mathrm{S}} \\
\frac{\partial^{2} U}{\partial S \partial V}=-\left(\frac{\partial P}{\partial S}\right)_{\mathrm{V}}=+\left(\frac{\partial T}{\partial V}\right)_{\mathrm{S}}
\end{gathered}
$$

These relations are valid at constant surface area $A$ and constant number of adsorbed charged species $N$. It is notable that the natural variables appear automatically in the denominators on the left-hand side. Further relations, in which changes occur by variation of the surface area $A$ or the number of adsorbed charged species $N$, are readily obtained by substitution, as we shall demonstrate below.

\section{The Electrochemical Potential}

Electrochemical potential is not the same thing as electrostatic potential.

Electrochemical potential is the total work (chemical and electrical) done in bringing a charged species (such as an electron or ion) from infinity to a specified location. Thus we write

$$
\bar{\mu}_{\mathrm{i}}=\mu_{\mathrm{i}}+z e \phi
$$

where $\bar{\mu}_{\mathrm{i}}$ is the electrochemical potential (J) of species $i, \mu_{\mathrm{i}}$ is the chemical potential (J) of species $i, z$ is the elementary charge number (dimensionless) of species $i, e$ is 
the elementary charge (C), and $\phi$ is the local electrostatic potential (Galvani potential). At thermodynamic equilibrium, it is the electrochemical potential that is constant across a system, not the electrostatic potential. In the special case of an uncharged species, $z=0$ and so $\bar{\mu}_{\mathrm{i}}=\mu_{\mathrm{i}}$.

Because it contributes to the Gibbs energy, the electrochemical potential can also be expressed as a partial derivative of the Gibbs energy (J) with respect to an infinitesimal increment of the number of charged species $N$ at constant $T$, and $P$.

$$
\bar{\mu}_{\mathrm{i}}=\left(\frac{\partial G}{\partial N}\right)_{\mathrm{T}, \mathrm{P}}
$$

Finally, and most importantly for the present work, we are interested in the case where the electrode potential $E$ is treated as an independent variable under the control of an experimenter. Assuming the electrode/solution interface is "ideally polarizable", then the chemical potentials in each bulk phase are constants, and therefore any change in electrode potential $E$ between the bulk phases necessarily induces a change in electrochemical potential $\bar{\mu}_{\mathrm{i}}$ of magnitude

$$
\partial \bar{\mu}_{\mathrm{i}}=z_{\mathrm{i}} e \partial E
$$

This equation is in full agreement with Eqs. (4.3.5) and (4.3.6) of Trasatti and Parsons [10].

In the vast majority of electrochemical systems, a change in electrode potential between an electrode and a solution has the effect of driving charge carriers across the electrode/solution interface. In the present work, however, we restrict attention to the special case of an "Ideally Polarizable Electrode", as defined by Koenig [11]. This is a two-phase system composed of an electronic conductor in contact with an ionic conductor, such that each type of charge carrier is confined to one phase only. In this situation, the transfer of charge carriers across the interface is impossible, and instead they simply pile up at the interface. As is well known, a few real electrodes (such as mercury and platinum) are nearly "ideally polarizable" at room temperature over certain ranges of applied potential in certain electrolyte solutions.

\section{The Gokhshtein Relations}

In his book "Surface Tension of Solids and Adsorption" (published in Russian) A. Ya. Gokhshtein introduced two new relations into surface thermodynamics, valid at constant temperature and pressure, having the form 


$$
+\left(\frac{\partial E}{\partial A}\right)_{\mathrm{Q}}=+\left(\frac{\partial \gamma}{\partial Q}\right)_{\mathrm{A}}
$$

and

$$
+\left(\frac{\partial \gamma}{\partial E}\right)_{\mathrm{A}}=-\left(\hat{q}+A \frac{\partial \hat{q}}{\partial A}\right)_{\mathrm{E}}
$$

Here $E$ is the electrode potential, $A$ is the surface area, $\gamma$ is the surface tension, $Q$ is the surface charge, and $\hat{q}$ is the surface charge density [6]. These relations were then used to analyse elastic changes in the surface of solids subject to electrochemical perturbations (the "estance" method). More recently, the logical status of G1 and G2 has been disputed in the scientific literature [12-21]. To help clarify these issues, I here provide a detailed derivation of the Gokhshtein Relations via the Gibbs Potential. [Gokhshtein himself derived his eponymous relations by means of the Landau and Helmholtz potentials.] It should also be noted that a short derivation of the Gokhshtein relations, based on an assumed form of the Gibbs Potential, has previously been published by Valincius [22]. The same author has also reported some very interesting experimental results [23].

For a system involving surface charge and surface tension, the mathematical analysis is rather complex. However, it can be greatly simplified if only two degrees of freedom are allowed. In order to contrive this situation, we proceed in the following way. First, we assume that only one species is adsorbed, so that the electrochemical potentials of all the other species are unaffected by the surface. Second, we assume that the solvent is inert, and that surface dipole effects are absent. This allows us to write an equation for the differential of the internal energy having only five degrees of freedom:

$$
\mathrm{d} U=T \mathrm{~d} S-P \mathrm{~d} V+\gamma \mathrm{d} A+\mu_{\mathrm{i}} \mathrm{d} N+\phi \mathrm{d} Q
$$

If we now recall Faraday's Law, which states that

$$
\frac{\mathrm{d} Q}{\mathrm{~d} N}=z e
$$

then

$$
\mathrm{d} Q=z e \mathrm{~d} N
$$

Eq. (34) is an equation of state that describes the coupling between charge number and species number for ions. Due to the fact that charge is always incarnated in 
matter, it is not possible to change the number of charges without also changing the number of charged species.

Now substituting for $\mathrm{d} Q$ yields,

$$
\mathrm{d} U=T \mathrm{~d} S-P \mathrm{~d} V+\gamma \mathrm{d} A+\left[\mu_{\mathrm{i}} \mathrm{d} N+z e \phi \mathrm{d} N\right]
$$

Finally, collecting the terms inside the bracket together, we can decrease the number of degrees of freedom by one:

$$
\mathrm{d} U=T \mathrm{~d} S-P \mathrm{~d} V+\gamma \mathrm{d} A+\left[\overline{\mathrm{i}_{\mathrm{i}}} \mathrm{d} N\right]
$$

where $\overline{\mu_{\mathrm{i}}}$ is the electrochemical potential of the adsorbed species. (This idea due to Guggenheim [24].)

Because entropy and volume are difficult variables to control, we next make the Legendre transform

$$
G=U-T S+P V
$$

This yields

$$
\mathrm{d} G=-S \mathrm{~d} T+V \mathrm{~d} P+\gamma \mathrm{d} A+\bar{\mu}_{\mathrm{i}} \mathrm{d} N
$$

At this stage we still have four degrees of freedom. However, it is not difficult to keep the temperature and pressure constant. In that case $\mathrm{d} T=0$ and $\mathrm{d} P=0$, and

$$
\mathrm{d} G=\gamma \mathrm{d} A+\bar{\mu}_{\mathrm{i}} \mathrm{d} N \quad(\text { at constant } T \text { and } P \text { ) }
$$

Thus we have achieved our goal of a system controlled by just two degrees of freedom $(A$ and $N)$. In summary, at constant temperature and pressure, the relevant thermodynamic potential is the Gibbs potential $G$, and the independent variables are the surface area $A$ and the number of adsorbed charged species $N$.

If we now recall that, for an ideally polarizable electrode/solution interface,

$$
\partial \bar{\mu}_{\mathrm{i}}=z_{\mathrm{i}} e \partial E
$$


where $\bar{\mu}_{\mathrm{i}}$ is the electrochemical potential of the charged species and $E$ is the electrode potential, then the corresponding Maxwell relations may be written (at constant $T$ and $P$ ),

$$
\begin{aligned}
& +\left(\frac{\partial E}{\partial A}\right)_{\mathrm{Q}}=+\left(\frac{\partial \gamma}{\partial Q}\right)_{\mathrm{A}} \\
& +\left(\frac{\partial \gamma}{\partial E}\right)_{\mathrm{A}}=-\left(\frac{\partial Q}{\partial A}\right)_{\mathrm{E}} \\
& -\left(\frac{\partial E}{\partial \gamma}\right)_{\mathrm{Q}}=+\left(\frac{\partial A}{\partial Q}\right)_{\gamma} \\
& +\left(\frac{\partial Q}{\partial \gamma}\right)_{\mathrm{E}}=+\left(\frac{\partial A}{\partial E}\right)_{\gamma}
\end{aligned}
$$

We see immediately that $\mathrm{M} 1$ is also the first Gokhshtein relation $\mathrm{G} 1$, and $\mathrm{M} 2$ is a generalization of the Lippmann equation. [The conventional Lippmann equation assumes that $(\partial Q / \partial A)_{\mathrm{E}}$ is a constant charge density, $q_{\mathrm{A}}$, even though this is not true in general.]

Defining a surface excess charge density $\hat{q}$ (which is not necessarily a constant independent of $A$ ) we have

$$
\hat{q}=\frac{Q(A)}{A}
$$

so that

$$
\partial Q=A \partial \hat{q}+\hat{q} \partial A
$$

Substituting into M2 yields

$$
+\left(\frac{\partial \gamma}{\partial E}\right)_{\mathrm{A}}=-\left(\hat{q}+A \frac{\partial \hat{q}}{\partial A}\right)_{\mathrm{E}}
$$

and we have now arrived at the second Gokhshtein relation G2. The final term is the modulus of surface excess charge density, and tells us how much the surface excess charge density varies with surface area at constant potential.

\section{Conclusions}


The terminology and definition of surface tension have been discussed. Under conditions of constant temperature and pressure, the surface tension has been defined as the partial derivative of the surface excess Gibbs energy with respect to an infinitesimal increment of surface area. This definition differs from the IUPAC definition [25], which states that surface tension is "the work required to increase a surface area divided by that area". In fact, the latter is the definition of the surface excess Gibbs energy density $\hat{G}(A)$.

In addition, a new formula for surface stress has been developed based upon a first order expansion of surface tension $\gamma(A)$ with respect to area.

Finally, the Gokhshtein relations have been derived from the Gibbs potential, based on the assumption that the electrode/solution interface is ideally polarizable. The Gokhshtein relations are thereby shown to be rigorous.

\section{Acknowledgements}

I thank two anonymous referees for suggesting improvements to the text.

\section{References}

[1] Anon (2013) Interface 22:28

[2] Frumkin AN, Bagotsky VS, lofa ZA, Kabanov BN (1952) Kinetika Elektrodnykh Protsessov. Moscow University Publishing House: Moscow

[3] Bagotsky VS (2012) Fuel Cells: Problems and Solutions. The Electrochemical Society Series 56 Wiley:Hoboken NJ

[4] Bagotsky VS (2005) Fundamentals of Electrochemistry. $2^{\text {nd }}$ Edition. The Electrochemical Society Series 44 Wiley:Hoboken NJ

[5] Rusanov Al (2012) Colloid Journal 74:136

[6] Gokhshtein AY (1976) Surface Tension of Solids and Adsorption, Nauka: Moscow (in Russian)

[7] Fletcher S (2005) Australian Journal of Chemistry 58:302

[8] Defay R, Prigogine I (1966) Surface Tension and Adsorption, Longmans: London.

[9] Shuttleworth R (1950) Proceedings of the Physical Society 63:444

[10] Trasatti S, Parsons R (1986) Journal of Electroanalytical Chemistry and Interfacial Electrochemistry 205:359 
[11] Koenig FO (1934) J Phys Chem 38:111

[12] Gutman EM (2011) Surface Science 605:1923

[13] Gutman EM (2012) J Solid State Electrochem 16:2283

[14] Gutman EM (2012) Surface Science 606:772

[15] Marichev VA (2010) Surface Science 604:458

[16] Marichev VA (2011) Surface Science 605:2097

[17] Marichev VA (2012) Protection of Metals and Physical Chemistry of Surfaces 48:27

[18] Kramer D, Weissmüller J (2007) Surface Science 601:3042

[19] Damaskin BB and Petrii OA (2011) J. Solid State Electrochem 15:1317

[20] Damaskin BB and Petrii OA (2012) J. Solid State Electrochem 16:2285

[21] Gokhshtein AY (2012) J Solid State Electrochem 16:3683

[22] Valincius G (1998) Langmuir 14:6307

[23] Valincius G (1999) Journal of Electroanalytical Chemistry 478:40

[24] Guggenheim EA (1929) J Phys Chem 33:842

[25] Nic M, Jirát J, Kosata B, Jenkins A (2006) IUPAC Compendium of Chemical Terminology (The "Gold Book"). International Union of Pure and Applied Chemistry. 